\title{
Synthesis and antimicrobial activities of new oxime carbamates of 3-aryl-2-thioquinazolin-4(3H)-one
}

\author{
SURESH S PATIL ${ }^{\mathrm{a}, *}$, SWATI D JADHAV ${ }^{\mathrm{a}}$ and M B DESHMUKH ${ }^{\mathrm{b}}$ \\ ${ }^{a}$ Organic Research Laboratory, Padmabhushan Dr. Vasantraodada Patil College, Tasgaon, \\ Dist. Sangli 416 312, India \\ ${ }^{\mathrm{b}}$ Department of Chemistry, Shivaji University, Kolhapur 416 004, India \\ e-mail: sanyujapatil@yahoo.com
}

MS received 4 September 2011; revised 24 April 2012; accepted 11 May 2012

\begin{abstract}
S-alkylation of 3-aryl-2-thioquinazolin-4(3H)-one (1) with chloroacetone gave 2-(propanonyl thio)-3-arylquinazol-4(3H)ones (2). Further, the treatment of compound (2) with hydroxylamine hydrochloride gave the corresponding oximes $(3)$ which on reaction with phenyl isocyanate in THF yielded corresponding oxime carbamates 4 . The synthesized compounds have been confirmed using IR and ${ }^{1} \mathrm{H}$ NMR, mass spectral data together with elemental analysis. All newly synthesized compounds have been tested for their antibacterial and antifungal activities.
\end{abstract}

Keywords. Thioquinazolinones; oximes; oxime carbamates; phenyl isocyanate; hydroxylamine hydrochloride.

\section{Introduction}

Natural products containing quinazolinone moiety possess a broad spectrum of biological activities, ${ }^{1,2}$ especially antimicrobial, ${ }^{3}$ antiulcer, ${ }^{4}$ pesticidal, ${ }^{5}$ antifungal, ${ }^{6,7}$ insecticidal, ${ }^{8}$ antibacterial, ${ }^{9}$ anticonvulsant, ${ }^{10,11}$ antithrombic, ${ }^{12}$ antitubercular, ${ }^{13}$ antitumour, ${ }^{14}$ etc. In this paper, we report a new route for the synthesis of 2-thio-3-aryl derivatives of quinazolinone.

\section{Experimental}

All ${ }^{1} \mathrm{H}$ NMR spectra were scanned on a Bruker A300 F-NMR spectrometer. IR spectra were recorded on a Perkin Elmer-783 spectrophotometer. All chemicals used were of AR grade and are used without further purification. Melting points are uncorrected and were measured on a DBK programmable melting point apparatus. Purity of the products was checked by TLC.

The starting compounds 1a-f were prepared by reported method. ${ }^{15}$

\subsection{3-(4-methylphenyl)-2-[(2-} oxopropyl)sulfanyl]quinazolin-4(3H)-one (2a)

The compound 1a $(2.84 \mathrm{~g}, 0.01 \mathrm{~mol})$ and chloroacetone $(0.93 \mathrm{~g}, 0.01 \mathrm{~mol})$ in dry acetone $(10 \mathrm{ml})$, to which

*For correspondence anhydrous potassium carbonate was added and reaction mixture refluxed for $18 \mathrm{~h}$ (monitored by TLC). The mixture was cooled and separated solid was extracted with $20 \mathrm{ml}$ ether. After removal of ether under reduced pressure gave solid, which was recrystallized from ethanol to furnish $2 \mathbf{a}$; Yield: $70 \%$; m.p. $100^{\circ} \mathrm{C}$. (Found: $\mathrm{C}, 66.65 ; \mathrm{H}, 4.95 ; \mathrm{N}, 8.69 \mathrm{C}_{18} \mathrm{H}_{16} \mathrm{O}_{2} \mathrm{~N}_{2} \mathrm{~S}$ requires: $\mathrm{C}, 66.64 ; \mathrm{H}, 4.97 ; \mathrm{N}, 8.64 \%)$; IR (KBr) $\mathrm{cm}^{-1}: 1718$ $(\mathrm{C}=\mathrm{O}), 1680$ (cyclic amide $\mathrm{C}=\mathrm{O}), 1615(\mathrm{C}=\mathrm{N}) ;{ }^{1} \mathrm{H}$ $\mathrm{NMR}\left(\mathrm{CDCl}_{3}\right): \delta 2.23\left(3 \mathrm{H}, \mathrm{s},-\mathrm{COCH}_{3}\right), 2.41(3 \mathrm{H}, \mathrm{s}$, $\left.\mathrm{Ar}-\mathrm{CH}_{3}\right), 3.91\left(2 \mathrm{H}, \mathrm{s}, \mathrm{S}-\mathrm{CH}_{2}\right), 7.21-8.10(8 \mathrm{H}, \mathrm{m}, \mathrm{ArH})$ ppm; MS (m/z): 324(M+), 309, 281, 267, 235(100\%), 233, 91, 43; ${ }^{13} \mathrm{C}$ NMR: $23.7\left(\mathrm{Ar}-\mathbf{C H}_{3}\right), 29.6\left(\mathbf{C H}_{3}-\right.$ $\mathrm{C}=\mathrm{O}), 37.4\left(\mathrm{~S}-\mathrm{CH}_{2}-\mathrm{C}=\mathrm{O}\right), 120.2\left(\mathrm{C}_{9}\right), 121.3\left(\mathrm{C}_{2^{\prime}}\right.$ and $\left.\mathrm{C}_{6^{\prime}}\right), 122.5\left(\mathrm{C}_{8}\right), 127.9\left(\mathrm{C}_{6}\right), 128.8\left(\mathrm{C}_{5}\right), 129.1\left(\mathrm{C}_{1^{\prime}}\right)$, $129.4\left(\mathrm{C}_{3^{\prime}}\right.$ and $\left.\mathrm{C}_{5^{\prime}}\right), 133.7\left(\mathrm{C}_{7}\right), 134.4\left(\mathrm{C}_{4^{\prime}}\right), 147.3\left(\mathrm{C}_{10}\right)$, $162.3\left(\mathrm{C}_{4}\right), 164.0\left(\mathrm{C}_{2}\right), 202.1\left(\mathrm{CH}_{3}-\mathrm{C}=\mathrm{O}\right)$.

Compounds $\mathbf{2 b}$ to $2 \mathbf{f}$ were prepared by using same method.

\subsection{2-[2-(Hydroxyimino)propyl]sulfanyl-3-(4- methylphenyl)quinazolin-4(3H)-one (3a)}

To a mixture of compound $2 \mathbf{a}(0.5 \mathrm{~g}, 0.0018 \mathrm{~mol})$ and hydroxylamine hydrochloride $(0.1 \mathrm{~g}, 0.0036 \mathrm{~mol})$ in ethanol $(10 \mathrm{ml})$, sodium hydroxide $(1 \mathrm{~g})$ was added slowly in a small portion at a time and constant stirring with cooling the reaction mixture. The same reaction mixture was refluxed further for about $10 \mathrm{~min}$, 
cooled and then poured into dil. $\mathrm{HCl}(50 \mathrm{ml})$. The separated solid was filtered, washed with water, and recrystallized from ethanol, Yield: $87 \%$, m. p. $268^{\circ} \mathrm{C}$. (Found: C, 63.75; H, 5.09; N, $12.39 \mathrm{C}_{18} \mathrm{H}_{17} \mathrm{O}_{2} \mathrm{~N}_{3} \mathrm{~S}$ requires: $\mathrm{C}, 63.70 ; \mathrm{H}, 5.05 ; \mathrm{N}, 12.38 \%) ; \mathrm{IR}(\mathrm{KBr}) \mathrm{cm}^{-1}$ : 3460-3340 (oxime -OH), 1672 (cyclic amide $\mathrm{C}=\mathrm{O}$ ), 1620 and $1612(\mathrm{C}=\mathrm{N}) \mathrm{cm}^{-1}$; ${ }^{1} \mathrm{H}$ NMR: $\delta 2.12(3 \mathrm{H}, \mathrm{s},-$ $\left.\mathrm{N}=\mathrm{C}-\mathrm{CH}_{3}\right), 2.32\left(3 \mathrm{H}, \mathrm{s}, \mathrm{Ar}-\mathrm{CH}_{3}\right), 4.16\left(2 \mathrm{H}, \mathrm{s}, \mathrm{S}-\mathrm{CH}_{2}\right)$, 7.03-8.11(8H, m, Ar-H), $10.42(1 \mathrm{H}, \mathrm{s},-\mathrm{OH}) \mathrm{ppm}$; MS (m/z): 339( $\left.\mathrm{M}^{+}\right), 324,308,248,235(100 \%), 104$, 91; ${ }^{13} \mathrm{C}$ NMR: $17.3,\left(\mathbf{C H}_{3}-\mathrm{C}=\mathrm{N}\right), 24.3\left(\mathrm{Ar}-\mathbf{C H}_{3}\right), 32.1$ $\left.\left(\mathrm{S}_{-} \mathrm{CH}_{2}\right)^{-}\right), 120.9\left(\mathrm{C}_{9}\right), 120.8\left(\mathrm{C}_{2^{\prime}}\right.$ and $\left.\mathrm{C}_{6^{\prime}}\right), 121.7\left(\mathrm{C}_{8}\right)$, $127.2\left(\mathrm{C}_{6}\right), 128.8\left(\mathrm{C}_{5}\right), 129.2\left(\mathrm{C}_{1^{\prime}}\right), 129.6\left(\mathrm{C}_{3^{\prime}}\right.$ and $\left.\mathrm{C}_{5^{\prime}}\right)$, $134.0\left(\mathrm{C}_{7}\right), 134.1\left(\mathrm{C}_{4^{\prime}}\right), 147.1\left(\mathrm{C}_{10}\right), 161.1\left(\mathrm{C}_{4}\right), 163.6$ $\left(\mathrm{C}_{2}\right), 169.0(\mathbf{C}=\mathrm{N}-\mathrm{OH})$.

Compounds $\mathbf{3 b}$ to $\mathbf{3 f}$ were prepared by operating same method.

\subsection{2-[2'-(O-phenylcarbamoylimino $)$ propylidene thio] 3-(p-methylphenyl) quinazolin-4(3H)one (4a)}

The equimolar mixture of $\mathbf{3 a}(0.20 \mathrm{~g}, 0.0006 \mathrm{~mol})$ and phenylisocynate $(0.07 \mathrm{~g}, 0.0006 \mathrm{~mol})$ in THF $(10 \mathrm{ml})$ was heated on a steam bath at $70^{\circ} \mathrm{C}$ for $2 \mathrm{~h}$, cooled and the separated solid was filtered and recrystallized from ethanol, Yield: $90 \%$, m. p. $235^{\circ} \mathrm{C}$. (Found: C, 65.52; $\mathrm{H}, 4.82 ; \mathrm{N}, 12.25 \mathrm{C}_{25} \mathrm{H}_{22} \mathrm{O}_{3} \mathrm{~N}_{4} \mathrm{~S}$ requires: $\mathrm{C}, 65.49 ; \mathrm{H}, 4.84 ; \mathrm{N}, 12.22 \%) ; \mathrm{IR}(\mathrm{KBr}) \mathrm{cm}^{-1}: 1710$ (carbamate $\mathrm{C}=\mathrm{O}$ ), 1676 (cyclic amide $\mathrm{C}=\mathrm{O}$ ), 1610 and $1612(\mathrm{C}=\mathrm{N}) \mathrm{cm}^{-1}$; ${ }^{1} \mathrm{H}$ NMR: $\delta 2.15(3 \mathrm{H}, \mathrm{s}$, $\left.-\mathrm{N}=\mathrm{C}-\mathrm{CH}_{3}\right), 2.35\left(3 \mathrm{H}, \mathrm{s}, \mathrm{Ar}-\mathrm{CH}_{3}\right), 4.12\left(2 \mathrm{H}, \mathrm{s}, \mathrm{S}-\mathrm{CH}_{2}\right)$, 7.20-8.11(13H, m, Ar-H), 8.61 (1H, s, -NH) ppm; MS $(\mathrm{m} / \mathrm{z}): 458\left(\mathrm{M}^{+}\right), 367,366,322(100 \%), 235,223,136$, 91, 77; ${ }^{13} \mathrm{C}$ NMR: 23.1, $\left(\mathbf{C H}_{3}-\mathrm{C}=\mathrm{N}\right), 24.3\left(\mathrm{Ar}-\mathbf{C H}_{3}\right)$, $24.9\left(\mathrm{~S}_{-} \mathrm{CH}_{2}-\right), 119.9\left(\mathrm{C}_{9}\right), 121.5\left(\mathrm{C}_{2^{\prime \prime}}\right.$ and $\left.\mathrm{C}_{6^{\prime \prime}}\right), 121.8$ $\left(\mathrm{C}_{2^{\prime}}\right.$ and $\left.\mathrm{C}_{6^{\prime}}\right), 122.7\left(\mathrm{C}_{8}\right), 124.6\left(\mathrm{C}_{4^{\prime \prime}}\right), 127.2\left(\mathrm{C}_{6}\right), 128.4$ $\left(\mathrm{C}_{5}\right), 127.2\left(\mathrm{C}_{1^{\prime}}\right), 129.0\left(\mathrm{C}_{3^{\prime \prime}}\right.$ and $\left.\mathrm{C}_{5^{\prime \prime}}\right), 129.3\left(\mathrm{C}_{3^{\prime}}\right.$ and $\left.\mathrm{C}_{5^{\prime}}\right), 133.9\left(\mathrm{C}_{7}\right), 134.0\left(\mathrm{C}_{4^{\prime}}\right), 135.8\left(\mathrm{C}_{1^{\prime \prime}}\right), 146.9\left(\mathrm{C}_{10}\right)$, $151.8(\mathrm{O}-\mathrm{C}=\mathrm{O}-\mathrm{NH}), 161.1\left(\mathrm{C}_{4}\right), 163.2\left(\mathrm{C}_{2}\right), 164.5$ $(-\mathrm{C}=\mathrm{N}-\mathrm{O})$.

Compounds $\mathbf{4 b}$ to $\mathbf{4 f}$ were prepared by operating same method.

\subsection{Antimicrobial activity}

The antimicrobial screening of synthesized compounds was carried out by paper disc diffusion method at 100 ppm against Gram + ve bacteria B. substilis, S. aureus and Gram - ve bacteria like E. coli, P. vulgaris. The antifungal activity of the compounds was assayed using fungal species Aspergillus niger and Phytophora. Standard antibacterial streptomycin and antifungal griseofulvin were also screened under similar condition for comparison.

The results indicate that the compounds $2 \mathbf{e}, \mathbf{2 f}, \mathbf{3 e}, \mathbf{3 f}$, $\mathbf{4 e}$ and $\mathbf{4 f}$ exhibited good antimicrobial activity against above bacteria and fungal species, while the compounds 2d, 3a, 3d and 4d have moderate antimicrobial activity against both Gram + ve and - ve bacteria and fungal species (table 1).

The generalization can be made from these observations that the compounds with substituent groups like $-\mathrm{Cl}$ and $-\mathrm{Br}$ enhance the antimicrobial activity than the other substituted compounds.

\subsection{Analytical data of synthesized compounds}

2.5a 3-(2-Methylphenyl)-2-[(2-oxopropyl)sulfanyl] quinazolin-4(3H)-one $(\mathbf{2 b})$ : Yield: $76 \%$; m.p. $100^{\circ} \mathrm{C}$; (Found: C, 66.65; H, 4.95; N, $8.69 \mathrm{C}_{18} \mathrm{H}_{16} \mathrm{O}_{2} \mathrm{~N}_{2} \mathrm{~S}$ requires: $\mathrm{C}, 66.64 ; \mathrm{H}, 4.97 ; \mathrm{N}, 8.64 \%)$; $\mathrm{IR}(\mathrm{KBr}) \mathrm{cm}^{-1}$ : $1718(\mathrm{C}=\mathrm{O}), 1676($ cyclic amide $\mathrm{C}=\mathrm{O}), 1612(\mathrm{C}=\mathrm{N})$; ${ }^{1} \mathrm{H} \mathrm{NMR}\left(\mathrm{CDCl}_{3}\right): \delta 2.51\left(3 \mathrm{H}, \mathrm{s},-\mathrm{COCH}_{3}\right), 2.42(3 \mathrm{H}$, s, $\left.\mathrm{Ar}-\mathrm{CH}_{3}\right), 4.91\left(2 \mathrm{H}, \mathrm{s}, \mathrm{S}-\mathrm{CH}_{2}\right), 7.03-8.11(8 \mathrm{H}, \mathrm{m}, \mathrm{Ar}-$ $\mathrm{H})$ ppm. ${ }^{13} \mathrm{C}$ NMR: $15.2\left(\mathrm{Ar}-\mathbf{C H}_{3}\right), 29.6\left(\mathbf{C H}_{3}-\mathrm{C}=\mathrm{O}\right)$, $37.6\left(\mathrm{~S}-\mathrm{CH}_{2}-\mathrm{C}=\mathrm{O}\right), 120.1\left(\mathrm{C}_{10}\right), 122.2\left(\mathrm{C}_{6^{\prime}}\right) 122.6$ $\left(\mathrm{C}_{8}\right), 124.4\left(\mathrm{C}_{4^{\prime}}\right), 125.8\left(\mathrm{C}_{5^{\prime}}\right), 127.6\left(\mathrm{C}_{6}\right), 128.2\left(\mathrm{C}_{3^{\prime}}\right)$, $128.9\left(\mathrm{C}_{5}\right), 135.1\left(\mathrm{C}_{2^{\prime}}\right), 135.6\left(\mathrm{C}_{1^{\prime}}\right), 136.9\left(\mathrm{C}_{7}\right), 147.4$ $\left(\mathrm{C}_{9}\right), 161.1\left(\mathrm{C}_{4}\right), 163.8\left(\mathrm{C}_{2}\right), 201.4\left(\mathrm{CH}_{3}-\mathrm{C}=\mathrm{O}\right)$.

2.5b 3-(3-Methylphenyl)-2-[(2-oxopropyl)sulfanyl] quinazolin-4(3H)-one $(\mathbf{2 c})$ : Yield: $81 \%$; m.p. $145^{\circ} \mathrm{C}$; (Found: C, 66.65; H, 4.95; N, $8.69 \mathrm{C}_{18} \mathrm{H}_{16} \mathrm{O}_{2} \mathrm{~N}_{2} \mathrm{~S}$ requires: $\mathrm{C}, 66.64 ; \mathrm{H}, 4.97 ; \mathrm{N}, 8.64 \%)$; IR $(\mathrm{KBr}) \mathrm{cm}^{-1}$ : $1720(\mathrm{C}=\mathrm{O}), 1674($ cyclic amide $\mathrm{C}=\mathrm{O}), 1612(\mathrm{C}=\mathrm{N})$; ${ }^{1} \mathrm{H} \mathrm{NMR}\left(\mathrm{CDCl}_{3}\right): \delta, 2.3\left(3 \mathrm{H}, \mathrm{s},-\mathrm{COCH}_{3}\right), 2.5(3 \mathrm{H}$, $\left.\mathrm{s}, \mathrm{Ar}-\mathrm{CH}_{3}\right), 4.0\left(2 \mathrm{H}, \mathrm{s}, \mathrm{S}-\mathrm{CH}_{2}\right), 7.1-8.0(8 \mathrm{H}, \mathrm{m}, \mathrm{Ar}-\mathrm{H})$ ppm. ${ }^{13} \mathrm{C}$ NMR: $23.7\left(\mathrm{Ar}^{-\mathrm{CH}_{3}}\right), 29.3\left(\mathrm{CH}_{3}-\mathrm{C}=\mathrm{O}\right), 36.9$ $\left(\mathrm{S}-\mathrm{CH}_{2}-\mathrm{C}=\mathrm{O}\right), 118.2\left(\mathrm{C}_{6^{\prime}}\right), 121.1\left(\mathrm{C}_{10}\right), 122.1\left(\mathrm{C}_{8}\right)$, $123.4\left(\mathrm{C}_{2^{\prime}}\right), 124.6\left(\mathrm{C}_{4^{\prime}}\right), 128.2\left(\mathrm{C}_{5^{\prime}}\right), 127.3\left(\mathrm{C}_{6}\right), 128.9$ $\left(\mathrm{C}_{5}\right), 135.2\left(\mathrm{C}_{1^{\prime}}\right), 138.8\left(\mathrm{C}_{3^{\prime}}\right), 134.9\left(\mathrm{C}_{7}\right), 147.9\left(\mathrm{C}_{9}\right)$, $162.0\left(\mathrm{C}_{4}\right), 164.1\left(\mathrm{C}_{2}\right), 200.4\left(\mathrm{CH}_{3}-\mathrm{C}=\mathrm{O}\right)$.

2.5c 3-(4-Methoxyphenyl)-2-[(2-oxopropyl)sulfanyl] quinazolin-4(3H)-one (2d): Yield: $78 \%$; m.p. $168^{\circ} \mathrm{C}$; (Found: C, 63.45; H, 4.67; N, $8.29 \mathrm{C}_{18} \mathrm{H}_{16} \mathrm{O}_{3} \mathrm{~N}_{2} \mathrm{~S}$ requires: $\mathrm{C}, 63.51 ; \mathrm{H}, 4.74 ; \mathrm{N}, 8.23 \%)$; IR $(\mathrm{KBr}) \mathrm{cm}^{-1}$ : $1720(\mathrm{C}=\mathrm{O}), 1676($ cyclic amide $\mathrm{C}=\mathrm{O}), 1610(\mathrm{C}=\mathrm{N})$; ${ }^{1} \mathrm{H} \mathrm{NMR}\left(\mathrm{CDCl}_{3}\right): \delta 2.13\left(3 \mathrm{H}, \mathrm{s},-\mathrm{COCH}_{3}\right), 3.24(3 \mathrm{H}$, $\left.\mathrm{s}, \mathrm{Ar}-\mathrm{OCH}_{3}\right), 3.80\left(2 \mathrm{H}, \mathrm{s}, \mathrm{S}-\mathrm{CH}_{2}\right), 7.21-8.12(8 \mathrm{H}, \mathrm{m}$, Ar-H) ppm. ${ }^{13} \mathrm{C}$ NMR: $29.9\left(\mathbf{C H}_{3}-\mathrm{C}=\mathrm{O}\right), 38.1\left(\mathrm{~S}_{-} \mathbf{C H}_{2}-\right.$ $\mathrm{C}=\mathrm{O}), 56.7\left(\mathrm{Ar}-\mathrm{OCH}_{3}\right), 114.4\left(\mathrm{C}_{3^{\prime}}\right.$ and $\left.\mathrm{C}_{5^{\prime}}\right), 120.7$ $\left(\mathrm{C}_{10}\right), 121.9\left(\mathrm{C}_{8}\right), 122.6\left(\mathrm{C}_{2^{\prime}}\right.$ and $\left.\mathrm{C}_{6^{\prime}}\right), 125.3\left(\mathrm{C}_{1^{\prime}}\right), 127.1$ 
Table 1. Antimicrobial screening data of the compounds 2,3 and 4 (diameter of the zones of inhibition in $\mathrm{mm}$ ).

\begin{tabular}{|c|c|c|c|c|c|c|c|}
\hline \multirow[b]{2}{*}{ Compounds } & \multirow[b]{2}{*}{$\mathrm{R}$} & \multicolumn{4}{|c|}{ Bacteria } & \multicolumn{2}{|c|}{ Fungi } \\
\hline & & E. coli & P. valgaris & B. subtilis & S. aureus & Aspergillus niger & Phytophora spp. \\
\hline $2 a$ & $\mathrm{p}-\mathrm{CH}_{3}$ & 12 & 14 & 12 & 14 & 13 & 14 \\
\hline $2 \mathbf{b}$ & $\mathrm{O}-\mathrm{CH}_{3}$ & 10 & 13 & 12 & 13 & 12 & 15 \\
\hline $2 c$ & $\mathrm{~m}-\mathrm{CH}_{3}$ & 12 & 13 & 11 & 11 & 13 & 14 \\
\hline $2 d$ & $\mathrm{p}-\mathrm{OCH}_{3}$ & 16 & 15 & 18 & 17 & 17 & 18 \\
\hline $2 \mathrm{e}$ & $\mathrm{p}-\mathrm{Cl}$ & 22 & 23 & 21 & 20 & 21 & 18 \\
\hline $2 f$ & $\mathrm{p}-\mathrm{Br}$ & 23 & 21 & 19 & 18 & 21 & 22 \\
\hline $\mathbf{3 a}$ & $\mathrm{p}-\mathrm{CH}_{3}$ & 16 & 15 & 17 & 15 & 15 & 11 \\
\hline $3 \mathbf{b}$ & $\mathrm{o}-\mathrm{CH}_{3}$ & 11 & 16 & 15 & 15 & 13 & 14 \\
\hline $3 c$ & $\mathrm{~m}-\mathrm{CH}_{3}$ & 09 & 12 & 11 & 10 & 12 & 13 \\
\hline $3 d$ & $\mathrm{p}-\mathrm{OCH}_{3}$ & 17 & 19 & 16 & 17 & 19 & 17 \\
\hline $3 \mathbf{e}$ & $\mathrm{p}-\mathrm{Cl}$ & 22 & 23 & 20 & 21 & 20 & 19 \\
\hline 3f & $\mathrm{p}-\mathrm{Br}$ & 21 & 22 & 21 & 22 & 23 & 24 \\
\hline $4 a$ & $\mathrm{p}-\mathrm{CH}_{3}$ & 14 & 16 & 15 & 13 & 14 & 12 \\
\hline $4 b$ & $\mathrm{O}-\mathrm{CH}_{3}$ & 14 & 13 & 17 & 14 & 15 & 15 \\
\hline $4 c$ & $\mathrm{~m}-\mathrm{CH}_{3}$ & 14 & 12 & 13 & 14 & 11 & 12 \\
\hline $4 d$ & $\mathrm{p}-\mathrm{OCH}_{3}$ & 18 & 19 & 16 & 18 & 17 & 19 \\
\hline $4 e$ & $\mathrm{p}-\mathrm{Cl}$ & 23 & 21 & 22 & 20 & 23 & 21 \\
\hline $4 f$ & $\mathrm{p}-\mathrm{Br}$ & 24 & 22 & 22 & 21 & 23 & 24 \\
\hline & & & & Standards & & & \\
\hline 1 & Streptomycin & 24 & 23 & 22 & 23 & - & - \\
\hline 2 & Griseofulvin & - & - & - & - & 25 & 23 \\
\hline
\end{tabular}

Activity: Good: above $20 \mathrm{~mm}$; moderate: $15-20 \mathrm{~mm}$; low: below $15 \mathrm{~mm}$.

$\left(\mathrm{C}_{6}\right), 128.2\left(\mathrm{C}_{5}\right), 134.1\left(\mathrm{C}_{7}\right), 147.2\left(\mathrm{C}_{9}\right), 156.1\left(\mathrm{C}_{4^{\prime}}\right)$, $162.3\left(\mathrm{C}_{4}\right), 163.2\left(\mathrm{C}_{2}\right), 201.5\left(\mathrm{CH}_{3}-\mathrm{C}=\mathrm{O}\right)$.

2.5d 3-(4-Chlorophenyl)-2-[(2-oxopropyl)sulfanyl] quinazolin-4(3H)-one (2e): Yield: $75 \%$; m.p. $180^{\circ} \mathrm{C}$; (Found: $\mathrm{C}, 59.20 ; \mathrm{H}, 3.84 ; \mathrm{N}, 8.10 \mathrm{C}_{17} \mathrm{H}_{13} \mathrm{O}_{2} \mathrm{~N}_{2} \mathrm{SCl}$ requires: C, 59.21; $\mathrm{H}, 3.80 ; \mathrm{N}, 8.12 \%)$; IR $(\mathrm{KBr}) \mathrm{cm}^{-1}$ : $1722(\mathrm{C}=\mathrm{O}), 1681$ (cyclic amide $\mathrm{C}=\mathrm{O}), 1615(\mathrm{C}=\mathrm{N})$; ${ }^{1} \mathrm{H}$ NMR $\left(\mathrm{CDCl}_{3}\right): \delta 2.31\left(3 \mathrm{H}, \mathrm{s},-\mathrm{COCH}_{3}\right), 3.90(2 \mathrm{H}$, s, $\left.\mathrm{S}-\mathrm{CH}_{2}\right), 7.10-8.10(8 \mathrm{H}, \mathrm{m}, \mathrm{ArH}) \mathrm{ppm} .{ }^{13} \mathrm{C}$ NMR: $29.7\left(\mathrm{CH}_{3}-\mathrm{C}=\mathrm{O}\right), 37.8\left(\mathrm{~S}-\mathrm{CH}_{2}-\mathrm{C}=\mathrm{O}\right), 120.1\left(\mathrm{C}_{10}\right)$, $122.9\left(\mathrm{C}_{8}\right), 123.1\left(\mathrm{C}_{2^{\prime}}\right.$ and $\left.\mathrm{C}_{6^{\prime}}\right), 127.8\left(\mathrm{C}_{6}\right), 129.1\left(\mathrm{C}_{5}\right)$, $129.7\left(C_{4^{\prime}}\right), 129.2\left(C_{3^{\prime}}\right.$ and $\left.C_{5^{\prime}}\right), 131.1\left(C_{1^{\prime}}\right), 134.1\left(C_{7}\right)$, $148.6\left(\mathrm{C}_{9}\right), 162.3\left(\mathrm{C}_{4}\right), 163.2\left(\mathrm{C}_{2}\right), 201.5\left(\mathrm{CH}_{3}-\mathrm{C}=\mathrm{O}\right)$.

2.5e 3-(4-Bromophenyl)-2-[(2-oxopropyl)sulfanyl] quinazolin-4(3H)-one (2f): Yield: $74 \%$; m.p. $183^{\circ} \mathrm{C}$, (Found: C, 52.44; H, 3.39; N, $7.24 \mathrm{C}_{17} \mathrm{H}_{13} \mathrm{O}_{2} \mathrm{~N}_{2} \mathrm{SBr}$ requires: $\mathrm{C}, 52.45 ; \mathrm{H}, 3.37 ; \mathrm{N}, 7.20 \%)$; IR $(\mathrm{KBr}) \mathrm{cm}^{-1}$ : $1716(\mathrm{C}=\mathrm{O}), 1680($ cyclic amide $\mathrm{C}=\mathrm{O}), 1612(\mathrm{C}=\mathrm{N})$; ${ }^{1} \mathrm{H} \mathrm{NMR}\left(\mathrm{CDCl}_{3}\right): \delta 2.11\left(3 \mathrm{H}, \mathrm{s},-\mathrm{COCH}_{3}\right), 4.02(2 \mathrm{H}$, s, $\left.\mathrm{S}-\mathrm{CH}_{2}\right), 7.22-8.16(8 \mathrm{H}, \mathrm{m}, \mathrm{ArH}) \mathrm{ppm} .{ }^{13} \mathrm{C} \mathrm{NMR}$ : $29.1\left(\mathrm{CH}_{3}-\mathrm{C}=\mathrm{O}\right), 37.1 \quad\left(\mathrm{~S}-\mathrm{CH}_{2}-\mathrm{C}=\mathrm{O}\right), 120.1\left(\mathrm{C}_{4^{\prime}}\right)$, $121.3\left(\mathrm{C}_{10}\right), 122.1\left(\mathrm{C}_{8}\right), 122.8\left(\mathrm{C}_{2^{\prime}}\right.$ and $\left.\mathrm{C}_{6^{\prime}}\right), 127.1\left(\mathrm{C}_{6}\right)$, 129.6( $\left.\mathrm{C}_{5}\right), 130.9\left(\mathrm{C}_{1^{\prime}}\right), 132.2\left(\mathrm{C}_{3^{\prime}}\right.$ and $\left.\mathrm{C}_{5^{\prime}}\right), 134.3\left(\mathrm{C}_{7}\right)$, $147.2\left(\mathrm{C}_{9}\right), 161.3\left(\mathrm{C}_{4}\right), 162.9\left(\mathrm{C}_{2}\right), 200.5\left(\mathrm{CH}_{3}-\mathrm{C}=\mathrm{O}\right)$.
$2.5 f \quad 2-[2-($ Hydroxyimino $)$ propyl] sulfanyl-3-(2methylphenyl)quinazolin-4(3H)-one $\quad(\mathbf{3 b})$ : Yield: 93\%, m.p. $242^{\circ} \mathrm{C}$; (Found: C, 63.72; H, 5.04; N, $12.37 \mathrm{C}_{18} \mathrm{H}_{17} \mathrm{O}_{2} \mathrm{~N}_{3} \mathrm{~S}$ requires: $\mathrm{C}, 63.70 ; \mathrm{H}, 5.05 ; \mathrm{N}$, 12.38\%); $\mathrm{IR}(\mathrm{KBr}) \mathrm{cm}^{-1}$ : 3500-3310 (oxime -OH), 1667 (cyclic amide $\mathrm{C}=\mathrm{O}), 1618$ and $1610(\mathrm{C}=\mathrm{N})$ $\mathrm{cm}^{-1} ;{ }^{1} \mathrm{H}$ NMR: $\delta 2.15\left(3 \mathrm{H}, \mathrm{s},-\mathrm{N}=\mathrm{C}-\mathrm{CH}_{3}\right), 2.30(3 \mathrm{H}$, s, $\left.\mathrm{Ar}-\mathrm{CH}_{3}\right), 4.10\left(2 \mathrm{H}, \mathrm{s}, \mathrm{S}-\mathrm{CH}_{2}\right), 7.12-8.13(8 \mathrm{H}, \mathrm{m}, \mathrm{Ar}-$ $\mathrm{H}), 10.20(1 \mathrm{H}, \mathrm{s},-\mathrm{OH})$ ppm. ${ }^{13} \mathrm{C}$ NMR: $16.2\left(\mathrm{Ar}-\mathrm{CH}_{3}\right)$, $22.6\left(\mathrm{CH}_{3}-\mathrm{C}=\mathrm{N}\right), 25.6\left(\mathrm{~S}-\mathrm{CH}_{2}-\mathrm{C}=\mathrm{N}\right), 121.1\left(\mathrm{C}_{10}\right)$, $121.2\left(\mathrm{C}_{6^{\prime}}\right), 121.8\left(\mathrm{C}_{8}\right), 124.1\left(\mathrm{C}_{4^{\prime}}\right), 126.7\left(\mathrm{C}_{5^{\prime}}\right), 127.3$ $\left(\mathrm{C}_{6}\right), 128.6\left(\mathrm{C}_{5}\right), 129.1\left(\mathrm{C}_{3^{\prime}}\right), 134.6\left(\mathrm{C}_{7}\right), 134.7\left(\mathrm{C}_{2^{\prime}}\right)$, $135.1\left(\mathrm{C}_{1^{\prime}}\right), 147.2\left(\mathrm{C}_{9}\right), 161.0\left(\mathrm{C}_{4}\right), 164.2\left(\mathrm{C}_{2}\right), 165.2$ $(\mathbf{C}=\mathrm{N}-\mathrm{OH})$.

$2.5 \mathrm{~g} \quad 2-[2-($ Hydroxyimino $)$ propyl] sulfanyl-3-(3methylphenyl)quinazolin-4(3H)-one (3c): Yield: $90 \%$, m. p. $246^{\circ} \mathrm{C}$; (Found: C, 63.74; H, 5.05; N, 12.35 $\mathrm{C}_{18} \mathrm{H}_{17} \mathrm{O}_{2} \mathrm{~N}_{3} \mathrm{~S}$ requires: $\mathrm{C}, 63.70 ; \mathrm{H}, 5.05 ; \mathrm{N}, 12.38 \%$ ); $\mathrm{IR}(\mathrm{KBr}) \mathrm{cm}^{-1}$ : 3495-3362 (oxime -OH), 1670 (cyclic amide $\mathrm{C}=\mathrm{O}), 1622$ and $1611(\mathrm{C}=\mathrm{N}) \mathrm{cm}^{-1} ;{ }^{1} \mathrm{H}$ NMR: $\delta 2.20\left(3 \mathrm{H}, \mathrm{s},-\mathrm{N}=\mathrm{C}-\mathrm{CH}_{3}\right), 2.25\left(3 \mathrm{H}, \mathrm{s}, \mathrm{Ar}-\mathrm{CH}_{3}\right), 4.12$ $\left(2 \mathrm{H}, \mathrm{s}, \mathrm{S}-\mathrm{CH}_{2}\right), 6.90-8.05(8 \mathrm{H}, \mathrm{m}, \mathrm{Ar}-\mathrm{H}), 10.30(1 \mathrm{H}$, s, -OH) ppm. ${ }^{13} \mathrm{C}$ NMR: $22.7\left(\mathrm{CH}_{3}-\mathrm{C}=\mathrm{N}\right), 24.3(\mathrm{Ar}-$ $\left.\mathrm{CH}_{3}\right), 25.3\left(\mathrm{~S}-\mathrm{CH}_{2}-\mathrm{C}=\mathrm{N}\right), 119.2\left(\mathrm{C}_{2^{\prime}}\right), 120.8\left(\mathrm{C}_{10}\right)$, $121.3\left(\mathrm{C}_{2^{\prime}}\right), 122.16\left(\mathrm{C}_{8}\right), 123.9\left(\mathrm{C}_{4^{\prime}}\right), 127.5\left(\mathrm{C}_{6}\right), 127.8$ 
$\left(\mathrm{C}_{5}\right), 128.7\left(\mathrm{C}_{3^{\prime}}\right), 133.2\left(\mathrm{C}_{1^{\prime}}\right), 134.3\left(\mathrm{C}_{7}\right), 138.2\left(\mathrm{C}_{5^{\prime}}\right)$, $147.2\left(\mathrm{C}_{9}\right), 162.0\left(\mathrm{C}_{4}\right), 164.2\left(\mathrm{C}_{2}\right), 165.4(\mathbf{C}=\mathrm{N}-\mathrm{OH})$.

2.5h 2-[2-(Hydroxyimino $)$ propyl $]$ sulfanyl-3-(4methoxyphenyl)quinazolin-4(3H)-one (3d): Yield: $90 \%$, m. p. $250^{\circ} \mathrm{C}$; (Found: C, 60.85; H, 4.87; N, 11.87 $\mathrm{C}_{18} \mathrm{H}_{17} \mathrm{O}_{3} \mathrm{~N}_{3} \mathrm{~S}$ requires: $\mathrm{C}, 60.83 ; \mathrm{H}, 4.82 ; \mathrm{N}, 11.82 \%$ ); $\mathrm{IR}(\mathrm{KBr}) \mathrm{cm}^{-1}$ : 3445-3310 (oxime -OH), 1668(cyclic amide $\mathrm{C}=\mathrm{O}), 1620$ and $1614(\mathrm{C}=\mathrm{N}) \mathrm{cm}^{-1} ;{ }^{1} \mathrm{H}$ NMR: $\delta 2.22\left(3 \mathrm{H}, \mathrm{s},-\mathrm{N}=\mathrm{C}-\mathrm{CH}_{3}\right), 3.45\left(3 \mathrm{H}, \mathrm{s}, \mathrm{Ar}-\mathrm{OCH}_{3}\right)$, $4.00\left(2 \mathrm{H}, \mathrm{s}, \mathrm{S}-\mathrm{CH}_{2}\right), 7.10-8.20(8 \mathrm{H}, \mathrm{m}, \mathrm{Ar}-\mathrm{H}), 10.62$ $(1 \mathrm{H}, \mathrm{s},-\mathrm{OH})$ ppm. ${ }^{13} \mathrm{C}$ NMR: $22.9\left(\mathrm{CH}_{3}-\mathrm{C}=\mathrm{N}\right), 26.1$ $\left(\mathrm{S}-\mathrm{CH}_{2}-\mathrm{C}=\mathrm{N}\right), 55.8\left(\mathrm{Ar}-\mathrm{OCH}_{3}\right), 115.1\left(\mathrm{C}_{3^{\prime}}\right.$ and $\left.\mathrm{C}_{5^{\prime}}\right)$, $121.2\left(\mathrm{C}_{10}\right), 121.6\left(\mathrm{C}_{2^{\prime}}\right.$ and $\left.\mathrm{C}_{6^{\prime}}\right), 122.2\left(\mathrm{C}_{8}\right), 125.1$ $\left(\mathrm{C}_{1^{\prime}}\right), 127.4\left(\mathrm{C}_{6}\right), 127.8\left(\mathrm{C}_{5}\right), 133.5\left(\mathrm{C}_{7}\right), 146.1\left(\mathrm{C}_{9}\right)$, $155.2\left(\mathrm{C}_{4^{\prime}}\right), 160.8\left(\mathrm{C}_{4}\right), 164.1\left(\mathrm{C}_{2}\right), 164.9(\mathbf{C}=\mathrm{N}-\mathrm{OH})$.

2.5i 2-[2-(Hydroxyimino)propyl]sulfanyl-3-(4chlorophenyl)quinazolin-4(3H)-one (3e): Yield: $84 \%$, m. p. $248^{\circ} \mathrm{C}$; (Found: C, 56.77; H, 3.90; N, $11.64 \mathrm{C}_{17} \mathrm{H}_{14} \mathrm{O}_{2} \mathrm{~N}_{3} \mathrm{SCl}$ requires: $\mathrm{C}, 56.74 ; \mathrm{H}, 3.92 ; \mathrm{N}$, 11.68\%); $\mathrm{IR}(\mathrm{KBr}) \mathrm{cm}^{-1}$ : 3483-3312 (oxime -OH), 1665 (cyclic amide $\mathrm{C}=\mathrm{O}), 1618$ and $1614(\mathrm{C}=\mathrm{N})$ $\mathrm{cm}^{-1}$; ${ }^{1} \mathrm{H}$ NMR: $\delta 2.25\left(3 \mathrm{H}, \mathrm{s},-\mathrm{N}=\mathrm{C}-\mathrm{CH}_{3}\right), 4.20(2 \mathrm{H}$, s, S-CH $\left.\mathrm{CH}_{2}\right), 7.11-8.21(8 \mathrm{H}, \mathrm{m}, \mathrm{Ar}-\mathrm{H}), 10.50(1 \mathrm{H}, \mathrm{s},-\mathrm{OH})$ ppm. ${ }^{13} \mathrm{C}$ NMR: $23.1\left(\mathrm{CH}_{3}-\mathrm{C}=\mathrm{N}\right), 25.5\left(\mathrm{~S}-\mathrm{CH}_{2}-\mathrm{C}=\mathrm{N}\right)$, $122.3\left(\mathrm{C}_{10}\right), 122.8\left(\mathrm{C}_{2^{\prime}}\right.$ and $\left.\mathrm{C}_{6^{\prime}}\right), 122.8\left(\mathrm{C}_{8}\right), 127.4\left(\mathrm{C}_{6}\right)$, 129.0 $\left(\mathrm{C}_{5}\right), 129.8\left(\mathrm{C}_{3^{\prime}}\right.$ and $\left.\mathrm{C}_{5^{\prime}}\right), 130.1\left(\mathrm{C}_{4^{\prime}}\right), 130.7\left(\mathrm{C}_{1^{\prime}}\right)$, $133.7\left(\mathrm{C}_{7}\right), 148.1\left(\mathrm{C}_{9}\right), 162.6\left(\mathrm{C}_{4}\right), 164.1\left(\mathrm{C}_{2}\right), 165.4$ $(\mathbf{C}=\mathrm{N}-\mathrm{OH})$.

2.5j 2-[2-(Hydroxyimino)propyl]sulfanyl-3-(4bromophenyl)quinazolin-4(3H)-one (3f): Yield: $87 \%$, m. p. $252^{\circ} \mathrm{C}$; (Found: C, 50.54; H, 3.47; N, $10.36 \mathrm{C}_{17} \mathrm{H}_{14} \mathrm{O}_{2} \mathrm{~N}_{3} \mathrm{SBr}$ requires: $\mathrm{C}, 50.50 ; \mathrm{H}, 3.49 ; \mathrm{N}$, 10.39\%); $\operatorname{IR}(\mathrm{KBr}) \mathrm{cm}^{-1}$ : 3500-3292 (oxime -OH), 1667 (cyclic amide $\mathrm{C}=\mathrm{O}), 1615$ and $1610(\mathrm{C}=\mathrm{N})$ $\mathrm{cm}^{-1}$; ${ }^{1} \mathrm{H}$ NMR: $\delta 2.22\left(3 \mathrm{H}, \mathrm{s},-\mathrm{N}=\mathrm{C}-\mathrm{CH}_{3}\right), 2.314 .20$ $\left(2 \mathrm{H}, \mathrm{s}, \mathrm{S}-\mathrm{CH}_{2}\right), 7.02-8.25(8 \mathrm{H}, \mathrm{m}, \mathrm{Ar}-\mathrm{H}), 10.64(1 \mathrm{H}, \mathrm{s}$, -OH) ppm. ${ }^{13} \mathrm{C}$ NMR: $22.9\left(\mathbf{C H}_{3}-\mathrm{C}=\mathrm{N}\right), 25.1\left(\mathrm{~S}_{-} \mathbf{C H}_{2}-\right.$ $\mathrm{C}=\mathrm{N}), 121.9\left(\mathrm{C}_{8}\right), 122.1\left(\mathrm{C}_{10}\right), 122.9\left(\mathrm{C}_{2^{\prime}}\right.$ and $\left.\mathrm{C}_{6^{\prime}}\right)$, $127.2\left(\mathrm{C}_{6}\right), 128.3\left(\mathrm{C}_{5}\right), 129.3\left(\mathrm{C}_{3^{\prime}}\right.$ and $\left.\mathrm{C}_{5^{\prime}}\right), 130.2\left(\mathrm{C}_{1^{\prime}}\right)$, $133.1\left(\mathrm{C}_{7}\right), 138.9\left(\mathrm{C}_{4^{\prime}}\right), 147.8\left(\mathrm{C}_{9}\right), 161.2\left(\mathrm{C}_{4}\right), 164.0$ $\left(\mathrm{C}_{2}\right), 164.3(\mathbf{C}=\mathrm{N}-\mathrm{OH})$.

$2.5 \mathrm{k} \quad 2-\left[2^{\prime}-(O-\right.$ phenylcarbamoylimino $)$ propylidene thio] 3-(2-methylphenyl) quinazolin-4(3H)one (4b): Yield: $82 \%$, m. p. $245^{\circ} \mathrm{C}$; (Found: C, 65.50; H, 4.85; $\mathrm{N}, 12.21 \mathrm{C}_{25} \mathrm{H}_{22} \mathrm{O}_{3} \mathrm{~N}_{4} \mathrm{~S}$ requires: $\mathrm{C}, 65.49 ; \mathrm{H}, 4.84$; $\mathrm{N}, 12.22 \%) ; \operatorname{IR}(\mathrm{KBr}) \mathrm{cm}^{-1}: 1713$ (carbamate $\mathrm{C}=\mathrm{O}$ ), 1672(cyclic amide $\mathrm{C}=\mathrm{O}), 1611$ and $1614(\mathrm{C}=\mathrm{N})$ $\mathrm{cm}^{-1} ;{ }^{1} \mathrm{H}$ NMR: $\delta 2.10\left(3 \mathrm{H}, \mathrm{s},-\mathrm{N}=\mathrm{C}-\mathrm{CH}_{3}\right), 2.30(3 \mathrm{H}$, $\left.\mathrm{s}, \mathrm{Ar}-\mathrm{CH}_{3}\right), 4.10\left(2 \mathrm{H}, \mathrm{s}, \mathrm{S}-\mathrm{CH}_{2}\right), 7.21-8.03(13 \mathrm{H}, \mathrm{m}$, Ar-H), 8.82(1H, s,-NH) ppm. ${ }^{13} \mathrm{C}$ NMR: 16.3 (Ar$\left.\mathbf{C H}_{3}\right), 22.1,\left(\mathbf{C H}_{3}-\mathrm{C}=\mathrm{N}\right), 24.6\left(\mathrm{~S}_{-} \mathbf{C H}_{2}-\right), 120.9\left(\mathrm{C}_{10}\right)$, $121.2\left(\mathrm{C}_{6^{\prime}}\right), 121.3\left(\mathrm{C}_{2^{\prime \prime}}\right.$ and $\left.\mathrm{C}_{6^{\prime \prime}}\right), 121.9\left(\mathrm{C}_{10}\right), 122.9$ $\left(\mathrm{C}_{8}\right), 124.1\left(\mathrm{C}_{4^{\prime \prime}}\right), 124.3\left(\mathrm{C}_{4^{\prime}}\right), 126.3\left(\mathrm{C}_{5^{\prime}}\right), 127.3\left(\mathrm{C}_{6}\right)$, $128.6\left(\mathrm{C}_{5}\right), 129.2\left(\mathrm{C}_{3^{\prime}}\right), 129.4\left(\mathrm{C}_{3^{\prime \prime}}\right.$ and $\left.\mathrm{C}_{5^{\prime \prime}}\right), 133.8$ $\left(\mathrm{C}_{7}\right), 134.8\left(\mathrm{C}_{2^{\prime}}\right), 135.2\left(\mathrm{C}_{1^{\prime}}\right), 135.9\left(\mathrm{C}_{1^{\prime \prime}}\right), 147.4\left(\mathrm{C}_{9}\right)$, $151.6(\mathrm{O}-\mathrm{C}=\mathrm{O}-\mathrm{NH}), 161.0\left(\mathrm{C}_{4}\right), 163.1\left(\mathrm{C}_{2}\right), 164.7$ $(-\mathrm{C}=\mathrm{N}-\mathrm{O})$.

$2.512-\left[2^{\prime}-(O-p h e n y l c a r b a m o y l i m i n o)\right.$ propylidene thio] 3-(3-methylphenyl) quinazolin-4(3H)one (4c): Yield: $83 \%$, m. p. $238^{\circ} \mathrm{C}$; (Found: C, 65.55; H, 4.80; $\mathrm{N}, 12.27 \mathrm{C}_{25} \mathrm{H}_{22} \mathrm{O}_{3} \mathrm{~N}_{4} \mathrm{~S}$ requires: $\mathrm{C}, 65.49 ; \mathrm{H}, 4.84$; $\mathrm{N}, 12.22 \%$ ); $\mathrm{IR}(\mathrm{KBr}) \mathrm{cm}^{-1}: 1715$ (carbamate $\mathrm{C}=\mathrm{O}$ ), 1675 (cyclic amide $\mathrm{C}=\mathrm{O}), 1610$ and $1615(\mathrm{C}=\mathrm{N})$ $\mathrm{cm}^{-1}$; ${ }^{1} \mathrm{H}$ NMR: $\delta 2.20\left(3 \mathrm{H}, \mathrm{s},-\mathrm{N}=\mathrm{C}-\mathrm{CH}_{3}\right), 2.30(3 \mathrm{H}$,
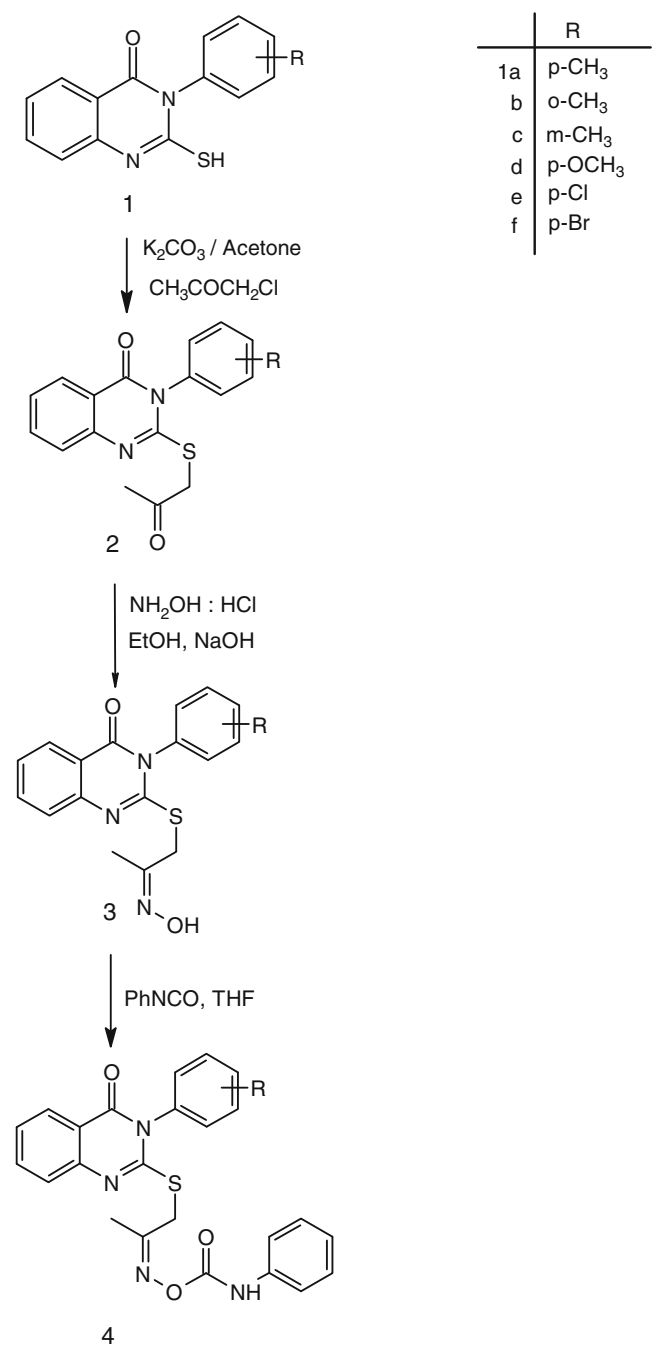

Scheme 1. Synthesis of oximes and oxime carbamates 3aryl-2-thioquinazolin-4(3H)-one. 
s, $\left.\mathrm{Ar}-\mathrm{CH}_{3}\right), 4.20\left(2 \mathrm{H}, \mathrm{s}, \mathrm{S}-\mathrm{CH}_{2}\right), 7.03-8.12(13 \mathrm{H}, \mathrm{m}$, Ar-H), $8.65(1 \mathrm{H}, \mathrm{s},-\mathrm{NH}) \mathrm{ppm} .{ }^{13} \mathrm{C}$ NMR: $22.5,\left(\mathbf{C H}_{3^{-}}\right.$ $\mathrm{C}=\mathrm{N}), 24.9\left(\mathrm{~S}_{-} \mathrm{CH}_{2^{-}}\right), 26.3\left(\mathrm{Ar}-\mathrm{CH}_{3}\right), 118.5\left(\mathrm{C}_{6^{\prime}}\right)$, $120.8\left(\mathrm{C}_{10}\right), 120.9\left(\mathrm{C}_{10}\right), 121.5\left(\mathrm{C}_{2^{\prime}}\right), 121.6\left(\mathrm{C}_{2^{\prime \prime}}\right.$ and $\left.\mathrm{C}_{6^{\prime \prime}}\right), 122.6\left(\mathrm{C}_{8}\right), 124.1\left(\mathrm{C}_{4^{\prime}}\right), 124.4\left(\mathrm{C}_{4^{\prime \prime}}\right), 127.7\left(\mathrm{C}_{6}\right)$, $128.2\left(\mathrm{C}_{5}\right), 128.7\left(\mathrm{C}_{5^{\prime}}\right), 129.1\left(\mathrm{C}_{3^{\prime \prime}}\right.$ and $\left.\mathrm{C}_{5^{\prime \prime}}\right), 133.2$ $\left(\mathrm{C}_{1^{\prime}}\right), 133.6\left(\mathrm{C}_{7}\right), 135.8\left(\mathrm{C}_{1^{\prime \prime}}\right), 138.3\left(\mathrm{C}_{3^{\prime}}\right), 147.2\left(\mathrm{C}_{9}\right)$, $151.9(\mathrm{O}-\mathrm{C}=\mathrm{O}-\mathrm{NH}), 160.8\left(\mathrm{C}_{4}\right), 163.0\left(\mathrm{C}_{2}\right), 164.6$ $(-\mathrm{C}=\mathrm{N}-\mathrm{O})$.

$2.5 \mathrm{~m} \quad 2-\left[2^{\prime}-(O-\right.$ phenylcarbamoylimino $)$ propylidene thio] 3-(4-methoxyphenyl) quinazolin-4(3H)one (4d): Yield: $85 \%$, m. p. $220^{\circ} \mathrm{C}$; (Found: C, 63.25; H, 4.69; $\mathrm{N}, 11.85 \mathrm{C}_{25} \mathrm{H}_{22} \mathrm{O}_{4} \mathrm{~N}_{4} \mathrm{~S}$ requires: $\mathrm{C}, 63.28 ; \mathrm{H}, 4.67 ; \mathrm{N}$, $11.81 \%)$; IR $(\mathrm{KBr}) \mathrm{cm}^{-1}: 1712($ carbamate $\mathrm{C}=\mathrm{O}), 1673$ (cyclic amide $\mathrm{C}=\mathrm{O}), 1616$ and $1610(\mathrm{C}=\mathrm{N}) \mathrm{cm}^{-1}$; ${ }^{1} \mathrm{H}$ NMR: $\delta 2.22\left(3 \mathrm{H}, \mathrm{s},-\mathrm{N}=\mathrm{C}-\mathrm{CH}_{3}\right), 3.35(3 \mathrm{H}, \mathrm{s}$, $\left.\mathrm{Ar}-\mathrm{OCH}_{3}\right), 4.20\left(2 \mathrm{H}, \mathrm{s}, \mathrm{S}-\mathrm{CH}_{2}\right), 7.21-8.14(13 \mathrm{H}, \mathrm{m}$,
$\mathrm{Ar}-\mathrm{H}), 8.65(1 \mathrm{H}, \mathrm{s},-\mathrm{NH})$ ppm. ${ }^{13} \mathrm{C}$ NMR: $22.8,\left(\mathrm{CH}_{3}-\right.$ $\mathrm{C}=\mathrm{N}), 25.3\left(\mathrm{~S}-\mathrm{CH}_{2}-\right), 54.8\left(\mathrm{Ar}-\mathrm{OCH}_{3}\right), 115.3\left(\mathrm{C}_{3^{\prime}}\right.$ and $\left.\mathrm{C}_{5^{\prime}}\right), 121.9\left(\mathrm{C}_{10}\right), 122.3\left(\mathrm{C}_{2^{\prime \prime}}\right.$ and $\left.\mathrm{C}_{6^{\prime \prime}}\right), 123.2\left(\mathrm{C}_{2^{\prime}}\right.$ and $\left.\mathrm{C}_{6^{\prime}}\right), 123.7\left(\mathrm{C}_{8}\right), 125.2\left(\mathrm{C}_{4^{\prime \prime}}\right), 125.2\left(\mathrm{C}_{1^{\prime}}\right), 126.2\left(\mathrm{C}_{6}\right)$, $127.4\left(\mathrm{C}_{5}\right), 129.7\left(\mathrm{C}_{3^{\prime \prime}}\right.$ and $\left.\mathrm{C}_{5^{\prime \prime}}\right), 133.4\left(\mathrm{C}_{7}\right), 135.3$ $\left(\mathrm{C}_{1^{\prime \prime}}\right), 147.9\left(\mathrm{C}_{9}\right), 152.3(\mathrm{O}-\mathrm{C}=\mathrm{O}-\mathrm{NH}), 156.9\left(\mathrm{C}_{4^{\prime}}\right)$, $160.1\left(\mathrm{C}_{4}\right), 163.1\left(\mathrm{C}_{2}\right), 165.5(-\mathrm{C}=\mathrm{N}-\mathrm{O})$.

$2.5 \mathrm{n} 2-\left[2^{\prime}-(O-p h e n y l\right.$ carbamoylimino $)$ propylidene thio] 3-(4-chlorophenyl) quinazolin -(3H)one (4e): Yield: $81 \%$, m. p. $245^{\circ} \mathrm{C}$; (Found: C, 60.21; H, 4.04; N, $11.75 \mathrm{C}_{24} \mathrm{H}_{19} \mathrm{O}_{3} \mathrm{~N}_{4} \mathrm{SCl}$ requires: $\mathrm{C}, 60.19 ; \mathrm{H}, 4.00 ; \mathrm{N}$, $11.70 \%) ; \mathrm{IR}(\mathrm{KBr}) \mathrm{cm}^{-1}: 1718$ (carbamate $\left.\mathrm{C}=\mathrm{O}\right), 1680$ (cyclic amide $\mathrm{C}=\mathrm{O}), 1620$ and $1615(\mathrm{C}=\mathrm{N}) \mathrm{cm}^{-1} ;{ }^{1} \mathrm{H}$ NMR: $\delta 2.17\left(3 \mathrm{H}, \mathrm{s},-\mathrm{N}=\mathrm{C}-\mathrm{CH}_{3}\right), 4.00\left(2 \mathrm{H}, \mathrm{s}, \mathrm{S}-\mathrm{CH}_{2}\right)$, 7.02-8.14(13H, m, Ar-H), 8.72(1H, s, -NH) ppm. ${ }^{13} \mathrm{C}$ NMR: $23.7\left(\mathbf{C H}_{3}-\mathrm{C}=\mathrm{N}\right), 25.1\left(\mathrm{~S}-\mathrm{CH}_{2}-\right), 121.4\left(\mathrm{C}_{2^{\prime \prime}}\right.$ and $\left.\mathrm{C}_{6^{\prime \prime}}\right), 122.2\left(\mathrm{C}_{10}\right), 123.8\left(\mathrm{C}_{2^{\prime}}\right.$ and $\left.\mathrm{C}_{6^{\prime}}\right), 123.9\left(\mathrm{C}_{8}\right)$, $124.9\left(\mathrm{C}_{4^{\prime \prime}}\right), 127.2\left(\mathrm{C}_{5}\right), 127.8\left(\mathrm{C}_{6}\right), 129.2\left(\mathrm{C}_{3^{\prime \prime}}\right.$ and<smiles>CC(C)=C(CCN)Sc1nc2ccccc2c(=O)n1-c1ccccc1</smiles>

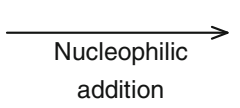<smiles>[R]c1ccc(-n2c(SCC(C)([O-])[NH2+]O)nc3ccccc3c2=O)cc1</smiles>

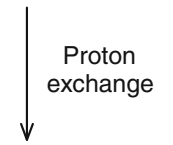

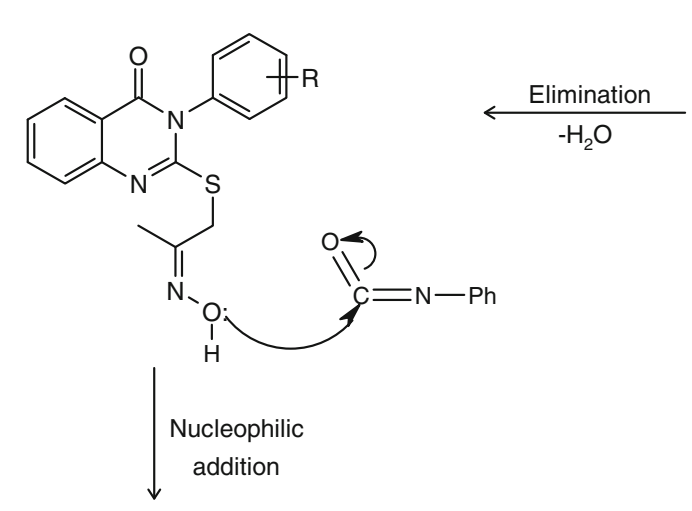<smiles>[Y]CCC(C)(O)C1(CSc2nc3ccccc3c(=O)n2-c2cccc([R])c2)CCN(O)C1</smiles>

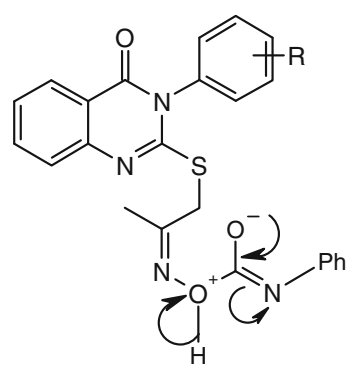<smiles>C/C(CSc1nc2ccccc2c(=O)n1-c1ccccc1)=N/OC(=O)Nc1ccccc1</smiles>

Scheme 2. Proposed mechanism for the synthesis of oximes and oxime carbamates 3-aryl-2-thioquinazolin-4(3H)-one. 
$\left.\mathrm{C}_{5^{\prime \prime}}\right), 129.3\left(\mathrm{C}_{3^{\prime}}\right.$ and $\left.\mathrm{C}_{5^{\prime}}\right), 130.2\left(\mathrm{C}_{1^{\prime}}\right), 130.9\left(\mathrm{C}_{4^{\prime}}\right), 133.9$

$\left(\mathrm{C}_{7}\right), 135.1\left(\mathrm{C}_{1^{\prime \prime}}\right), 147.4\left(\mathrm{C}_{9}\right), 151.2(\mathrm{O}-\mathrm{C}=\mathrm{O}-\mathrm{NH})$, $160.6\left(\mathrm{C}_{4}\right), 164.0\left(\mathrm{C}_{2}\right), 164.7(-\mathrm{C}=\mathrm{N}-\mathrm{O})$.

$2.50 \quad 2-\left[2^{\prime}-(O-p h e n y l c a r b a m o y l i m i n o)\right.$ propylidene thio] 3-(4-bromophenyl) quinazolin-4(3H)one (4f): Yield: $78 \%$, m. p. $271^{\circ} \mathrm{C}$; (Found: C, $55.09 ; \mathrm{H}, 3.65 ; \mathrm{N}$, $10.75 \mathrm{C}_{24} \mathrm{H}_{19} \mathrm{O}_{3} \mathrm{~N}_{4} \mathrm{SBr}$ requires: $\mathrm{C}, 55.07 ; \mathrm{H}, 3.66 ; \mathrm{N}$, $10.70 \%$ ); $\mathrm{IR}(\mathrm{KBr}) \mathrm{cm}^{-1}: 1720$ (carbamate $\left.\mathrm{C}=\mathrm{O}\right), 1679$ (cyclic amide $\mathrm{C}=\mathrm{O}), 1615$ and $1619(\mathrm{C}=\mathrm{N}) \mathrm{cm}^{-1} ;{ }^{1} \mathrm{H}$ NMR: $\delta 2.20\left(3 \mathrm{H}, \mathrm{s},-\mathrm{N}=\mathrm{C}-\mathrm{CH}_{3}\right), 3.90\left(2 \mathrm{H}, \mathrm{s}, \mathrm{S}-\mathrm{CH}_{2}\right)$, 7.12-8.04(13H, m, Ar-H), $8.52(1 \mathrm{H}, \mathrm{s},-\mathrm{NH}) \mathrm{ppm} .{ }^{13} \mathrm{C}$ NMR: 23.2, $\left(\mathbf{C H}_{3}-\mathrm{C}=\mathrm{N}\right), 24.8\left(\mathrm{~S}_{-} \mathbf{C H}_{2}-\right), 120.3\left(\mathrm{C}_{10}\right)$, $121.1\left(\mathrm{C}_{2 \text { ? }}\right.$ and $\left.\mathrm{C}_{6^{\prime \prime}}\right), 123.2\left(\mathrm{C}_{2^{\prime}}\right.$ and $\left.\mathrm{C}_{6^{\prime}}\right), 123.5\left(\mathrm{C}_{8}\right)$, $124.7\left(\mathrm{C}_{4^{\prime \prime}}\right), 127.6\left(\mathrm{C}_{6}\right), 126.9\left(\mathrm{C}_{5}\right), 128.9\left(\mathrm{C}_{3^{\prime \prime}}\right.$ and $\left.\mathrm{C}_{5^{\prime \prime}}\right), 129.1\left(\mathrm{C}_{3^{\prime}}\right.$ and $\left.\mathrm{C}_{5^{\prime}}\right), 130.0\left(\mathrm{C}_{1^{\prime}}\right), 120.4\left(\mathrm{C}_{4^{\prime}}\right), 133.6$ $\left(\mathrm{C}_{7}\right), 134.9\left(\mathrm{C}_{1^{\prime \prime}}\right), 147.6\left(\mathrm{C}_{9}\right), 150.8(\mathrm{O}-\mathrm{C}=\mathrm{O}-\mathrm{NH})$, $160.2\left(\mathrm{C}_{4}\right), 163.8\left(\mathrm{C}_{2}\right), 164.2(-\mathrm{C}=\mathrm{N}-\mathrm{O})$.

\section{Results and discussion}

3-Aryl-2-thioquinazolin-4(3H)-one 1a-f were used as a key starting material for the synthesis of oximes 3a-f and oxime carbamates 4a-f (scheme 1). First, 3aryl-2-thioquinazolin-4(3H)-one 1a-f were S-alkylated with chloroacetone in the presence of catalytic amount of potassium carbonate to give 2-(propanonyl thio)3 -arylquinazol-4(3H)ones $\mathbf{2 a - f}$. The formation compounds 2 have been explained by the appearance of bands at $1716-1722 \mathrm{~cm}^{-1}$ due to acyclic $>\mathrm{C}=\mathrm{O}$ in IR spectrum and two singlet encountered at $\delta, 2$ to 3 and 3 to 4 due to the $-\mathrm{COCH}_{3}$ and $-\mathrm{S}-\mathrm{CH}_{2}$ in the ${ }^{1} \mathrm{H}$ NMR spectrum, respectively. All compounds are obtained in high purity with good yield.

Compounds 2 on nucleophilic addition of hydroxylamine hydrochloride followed by elimination of water furnished oximes (3) (scheme 2). The formation of oximes 3 have been established by the appearance of bands at $3500-3292 \mathrm{~cm}^{-1}$ and disappearance of bands at $1716-1722 \mathrm{~cm}^{-1}$ in its IR spectrum due to $-\mathrm{OH}$ of and acyclic carbonyl, respectively. The formation of $\mathbf{3}$ were also supported by observing additional down field singlets at $\delta, 10.4-10.7 \mathrm{ppm}$ due to $-\mathrm{OH}$ protons in ${ }^{1} \mathrm{H}$ NMR spectrum.
The compounds $\mathbf{3}$ were transformed into their corresponding oxime carbamates 4 by reacting them with phenyl isocyanate in THF. The disappearance of IR bands at $3500-3292 \mathrm{~cm}^{-1}$ and ${ }^{1} \mathrm{H}$ NMR signals due to $-\mathrm{OH}$ protons confirmed their formation.

\section{Conclusion}

In this work, we have reported new oxime carbamate derivatives of 3-Aryl-2-thioquinazolin-4(3H)-one which are characterized by IR, PMR and ${ }^{13} \mathrm{C}$ NMR spectral analysis. Synthesized compounds are screened for their antifungal and antibacterial activity. These compounds show good activity against Gram + ve and -ve bacteria and fungal species.

\section{References}

1. Deshmukh M B, Patil S, Patil S S and Jadhav S D 2010 Indian J. Pharm. Sci. $\mathbf{7 2} 500$

2. Ammar Y A, El-Sharief A M Sh, Zahran M A, Ali A H and El-Gaby M S A 2001 Molecules 6267

3. El-Sharief A M Sh, Ammar Y A, Zahram M A and Ali A H 2002 J. Chem. Res. 5205

4. Patil A, Ganguly S and Suran S 2010 J. Chem. Sci. 22 443

5. Sen Gupta A K and Pandey A K 1989 Pesticide Sci. 26 41

6. Bennur S C, Talawar M B, Laddi U V, Somannavar Y S, Badigear V V and Virupakshaiah H M 1997 Indian J. Heterocycl. Chem. 739

7. Ghorab M M 2000 Farmaco 55249

8. Singh T, Sharma S, Kishore V and Shrivastava K A 2006 Indian J. Chem. 45B 2558

9. Singh S, Dave U and Parikh A R 1994 J. Indian Chem. Soc. 159

10. Parmar S S, Chaturvedi A K, Chaudhari A and Brumlene S J 1974 J. Pharm. Sci. 63356

11. Ghany A E A and Mohammed H A W 2003 Acta. Pharm. 53127

12. Demer J P, Sulsky R and Klaubert D H 1989 J. Heterocycl. Chem. 261535

13. Kunes J, Bazant J, Pour M, Waisser K, Slosarek M and Janota J 2000 Farmaco 55725

14. Deetz M J, Malerich J P, Beatty A M and Smith B D 2001 Tet. Lett. 421851

15. Deshmukh M B, Deshmukh D S and Shirke S D 1997 J. Indian Chem. Soc. $\mathbf{7 4} 422$ 